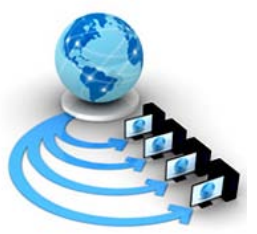

Volume 9, No. 2, March-April 2018

\title{
INTELLIGENT AUTOMATED IOT BASED IRRIGATION SYSTEM
}

\author{
Abhinav Jain \\ Department of Computer Science and Engineering, \\ Vellore Institute of Technology, \\ Vellore, India
}

\author{
Selvakumar K. \\ Department of Computer Science and Engineering, \\ Vellore Institute of Technology, \\ Vellore, India
}

\begin{abstract}
In the developing digital world, wireless sensor network helps in advance systems. The work follows the fundamental idea which is used to study the flow of data such as water flow, remove obstacles, and provide medicines based on crop fertilization stages during a transmission by use of wireless sensor network and monitoring system. The work is mainly focused on smart agriculture system and also provide the highly qualified crop yield by development in an automated precision agriculture monitoring system and also monitoring the quality of crop fertilization in stage by stage growth process by sensing / intimating flow of data based on crop fertilization varieties such as long duration varieties, medium duration varieties and short duration varieties for scheming standard parameters like humidity, temperature, soil moisture based on fertilization stages. Single-chip microcontrollers furnished with remote handsets are picking up ubiquity in brilliant home computerization considering their inherent assets, low power utilization, measure, manage the cost of capacity and sturdiness. Innovative work experts are grabbing the chance to outline and coordinate more capacities and administrations for savvy home checking and control systems using such microcontrollers. This project exhibits a wireless water irrigation system for a smart home garden that can be incorporated with existing savvy home control frameworks.
\end{abstract}

Keywords: Automated Irrigation System, Sensors, Crops, Soil Moisture, Humidity, Temperature, GSM, Water Pump

\section{INTRODUCTION}

I belong to a place called Punjab which has been known for agricultural goods, cycle industries and textiles. I myself being a part of agriculture industry saw people using old ways of farming, and if someone starts new technology that is still not fully mechanized. People have started drip irrigation, but that is something which you must switch on when they felt like, leading to increase in man power and being time bound. The overview of this work is about smart agriculture system and also provide the highly qualified crop yield by the using the ZigBee technology in WSNs and also monitoring the quality of crop fertilization in stage by stage growth process by sensing/intimating flow of data based on crop fertilization varieties such as long duration varieties, medium duration varieties and short duration varieties for controlling classic parameters like temperature, humidity, soil moisture based on fertilization stages.

Natural observing framework in agribusiness which is utilized to control the conservative method for supplement supply that will create the best harvest condition, increment the generation proficiency while diminishing expense. The constant data of this field will give a strong base to agriculturists which help to change procedures whenever. Natural observing framework in agribusiness which is utilized to control the conservative method for supplement supply that will create the best harvest condition, increment the generation proficiency while diminishing expense. The constant data of this field will give a strong base to agriculturists which help to change procedures whenever. WSN will reuse the information accumulation in agrarian research. Notwithstanding, there have been few investigates on the uses of WSN for agriculture.

The sensors are used for measuring temperature, humidity, soil moisture and intimate for remove obstacles and provide medicines based on crop fertilization stage that can be helps the farmers to maintain the crop production based on the crop fertilization varieties.

\section{RELATED WORK}

Many research papers have been published on this automated irrigation system using Microcontrollers, Arduino, Raspberry PI and few sensors.

The summary of the research papers taken for the survey are as follows:

[1] Agriculture majorly affects financial system of the state. Bundle of studies been finished in robotizing the water gadget structure by means of making utilization of remote sensor and bendy figuring. also, contemplates been done in using device becoming more acquainted with in agrarian system too as of now "machine to device (M2M)" correspondence is a creating development which lets in gadgets, things et cetera to hold among each exceptional and convey data to Server or Cloud through the inside group. So in like way, we appropriate here have built up a savvy IoT principally based totally programmed Irrigation system in which sensor records alluding to soil clamminess and temperature stuck and in like way KNN (approve Nearest Neighbor) depiction machine picking up learning of depend wide assortment dispatched for keeping separated the sensor certainties for evaluate towards flooding the earth with water. that is a totally programmed wherein units pass on among themselves and take a gander at the concentrate in inundating. This has been propelled utilizing low cost implanted gadgets like Arduino Uno, Raspberry PI.

[2] India's population is increasing day by day then once 25-30 years there'll be significant issue of food, therefore the development of agriculture is important. Today, the agriculturists locale unit overflowing with the absence of storms and deficiency of water. the most objective of this paper is to convey a customized water framework system in 
this way saving time, cash and impact of the agriculturist. The standard home arrive water framework strategies require manual mediation. With the machine-controlled advance of water structure the human mediation will be diminished. At whatever point there's an adjustment in temperature, stickiness, wetness and $\mathrm{pH}$ level of the condition these sensors sense the enhancements and offers an intruder with banner to the ARDUINO CONTROLLER. By then it will be exchanged to the IOT (Internet of Things) advancement so agriculturist will screen and association the water system from wherever.

[3] The moved headway in remote sensor frameworks can be used as a piece of checking distinctive parameters in cultivating. Considering choppy standard scrambling of rain water, it is to a mind-boggling diploma difficult for agriculturists to display screen and manage the automobile of water to agribusiness field within the entire residing course of motion or as showed with the aid of the want of the yield. there's no wonderful water framework system for every surroundings situation, soil structure and assembling of yields social solicitations. Agriculturists continue through enormous budgetary troubles by using virtue of incorrect need of air and blended up water shape philosophy. in this super situation, with the distinction in downsized sensor gadgets joined with faraway advances, it's far feasible remotely display screen parameters, as an instance, dampness, temperature and stickiness. in this paper it's miles proposed to setup, make and complete a far-flung sensor orchestrate related with a crucial attention the use of ZigBee, which therefore is related to a primary monitoring Station (CMS) through general Packet Radio carrier (GPRS) or international system for mobile (GSM) tendencies. The shape moreover receives worldwide Positioning device (GPS) parameters associated with the field and sends them to a vital looking station. This shape is depended on to assist farmers in evaluating soil situations and act in like manner.

[4] A mechanized water irrigation system was made to update water use for agrarian harvests. The structure has a surpassed on faraway machine of soil-wet state and temperature sensors set inside the root district of the verdure. In like way, a zone unit handles sensor data, triggers actuators, and transmits certainties to a web utility. An investigate was made with confine estimations of temperature and soil dousing that wound up adjusted into a humbler scale controller-based access to administer water hint. The shape was controlled by methods for picture voltaic sheets and had a duplex correspondence relate in setting of a versatile web interface that considered measurements exam and water framework criticalness to be adjusted through a website page. The robotized shape become attempted in a cautious trade area for 136 days and water spare sources of up to ninety\% disconnected and general water gadget practices of the provincial quarter had been pro. 3 duplicates of the robotized shape were used sensibly in diverse spots for eighteen months. because of its essentialness discretion and irrelevant exertion, the structure can be fundamental in water obliged topographically compelled zones.

[5] Agriculture plays the important role in INDIAN economy. The most important thing which is needed for the irrigation is water. This proposal discussed about how the water can be efficiently used for agriculture. According to this proposal Real Time Clock(RTC) is used to control the motor in the real time. First the ON time and OFF time of the motor is send from the android application via GPRS modem. Once the ON and OFF timings are reached the controller, the motor continuously starts and stops in that time interval in the by using RTC. Temperature and moisture values of the irrigation area are continuously monitored, and the values are send to the android application via GPRS modem. If the temperature and moisture values are beyond the certain limit an alert SMS will be send to the farmer. If the farmer wishes to control the motor remotely it is possible by pressing the ON and OFF button given in the android application.

[6] The earth sogginess sensor gives the banner which is comparing to the moistness level in the soil which is then differentiated and the breaking point regard that is gotten by investigating the diverse soil and also the specific sort of the items, the result by then procured is being supported into the Arduino scaled down scale controller which is related with the propelled cell phone, using which the information concerning the suddenness substance can be brought and which moreover acts like a remote control which consolidate murdering ON/the motor(switching). Trading is the technique that is used, the soil is attempted at various moistness levels. Discussed on the Chennai surges which was happened because of absence of good judgment of urban zones and there was encroachment aground. This remote-controlled organization can be used during the trademark upheavals like surges and silly precipitation. The water framework pumps close by the valve system can be used to control the water stream and likewise the course of stream. Regardless, the measure of the water stream can be controlled with the trading of the pump motors, the heading of the water stream can in like manner be coordinated which engages the discharging and letting the water from the estate fields. Finally, by invigorating the above system we can pass on in the progressing agriculture applications.

[7] In the system uses ease home-made clamminess sensor which is exceptionally correct. This home-made sensor uses a square of thermo-col nearby two long copper wires which are inserted at a settled detachment which acts like a sensor. The wires are maintained because of the sticks keeping the true objective to refrain from bowing of the wires when implanted into the soil. In like manner, distinctive sensors are being used to assemble the extent of the identifying contraption. The two wires set in the earth shape variable security which is then connected with the voltage divider. Arduino by then gets the voltage with respect to the security. This structure uses two procedures which are discussed as takes after. In the foremost procedure the case of soil the moistness is attempted by changing the measure of water content and the characteristics are recorded. Three zones of conductivity are described which are appeared by the three LED'S. If the red LED shimmers, by then it exhibits that the soil is dry also suggested as the dry zone. If the green LED sparkles on the PCB, it shows that the clamminess content is up to the level and does not require any more water. The third zone is the one in which the earth has more water content than the run of the mill. The second system deals with a comparative case of soil taken in a holder of certain stature and the moistness at different levels of the earth is being attempted, three sensors are being installed at three profundities. The yield of the three sensors is given as a basic data A0, A1, A2 to the Arduino board. The LED's can be used 
as a pointer of the notice if the water level is too low or too high. The experts are-insignificant exertion, capable. The calculation of the moisture at different levels fathoms that when and how much water is required for the base of the reap.

[8] In this author have given the purposes of enthusiasm of modernized water framework systems using the ESP8266 Wi-Fi module. Clever water framework structure should be completed in India as it is the second greatest exporter of rice. Rice on the other hand requires bounteous measures of water and in this country where there is lack of water, there must be a system to deal with the supply of water spilling into the fields. This structure includes contrasting sensors-moisture sensor, temperature sensor and the water stream sensor. The rule limit of the temperature sensor is that it records the temperature of the yields, since they are temperature fragile and if the temperature of the items outperforms a purpose of control the agriculturist can switch on the sprinkler to chill it off. This system can be open reliably and $24 * 7$ of openness lays on a more critical favored point of view. The water stream sensor has a pinwheel sensor which can evaluate the measure of water that has been gone through it. It furthermore has a little magnet associated that can record the conditions the pinwheel has turned. The beats are recorded which can show the measure of water that is traveling through it. Soil sogginess sensor uses the dielectric permittivity. Here the dielectric permittivity is just the limit of water content in the earth. The sensor makes the voltage relating to the water substance of the earth and the clamminess is being evaluated. All the control occurs by using the site. The key great position of using this structure is that it can be executed in the farms and cultivating territories like those of the rice trims in India with an insignificant exertion. Even though the inconvenience fuses the utilization of site, which can be hacked by anybody and the system glitches. Moreover, every one of the agriculturists won't be familiar with the utilization of the site and besides, they can't hold up under its cost.

\section{SYSTEM ANALYSIS AND DESIGN}

To gauge the status of the soil humidity sensor, water level sensor and moisture sensor has been utilized. The moisture sensor is utilized to gauge the volumetric water content in the soil. The humidity sensor measures the measure of water vapor noticeable all around. The water level sensor measures the water level of the soil. There by fulfilling the requirements of the earth. The whole structure contains sensors which screens the condition of the earth. The signs are send to the microcontroller. On the off chance that there is any nonappearance of water level in the soil, the microcontroller guides the water to the pined for level by utilizing engine. The coveted level is surveyed by sensors. The data about the condition of the soil is sent to the database. This information is then contrasted with standard edge esteems to check if the yield is in typical condition. If there is a contrast, then the message is sent to the person in charge.

Measurement of parameters: Calculation of all the constraints such as humidity, moisture in farm is vital to predict the selection of the next crop which a farmer should cultivate, also take decisions for betterment of the current yield. Fetching of Data: Sensors are estimating the parameters and those parameters ought to need to get for additionally process like information mining. Estimating and Fetching information are the nuts and bolts practical prerequisites of this framework.
Manipulation of Data: After collecting the data, this data is manipulated or checked according to pre-defined standards.

Crop selection: Based on the collected data, the farmer can take necessary actions. Detection of movements: Since farms are spread over a large area, it is important to detect any animal movement to protect the crops.

Notification on Display: All the readings and findings are notified to the farmer via cloud and the information is also notified on the LCD.

Perspective: India has huge population of agriculturists and in addition India's GDP is based very from the pay earned through cultivating. Cultivating is the texture of public and it is the fundamental monetary movement. Cultivating influences the nation as far as social and political soundness. To expand the profitability of our nation it is important make our agriculture area more robotization and digitalized.

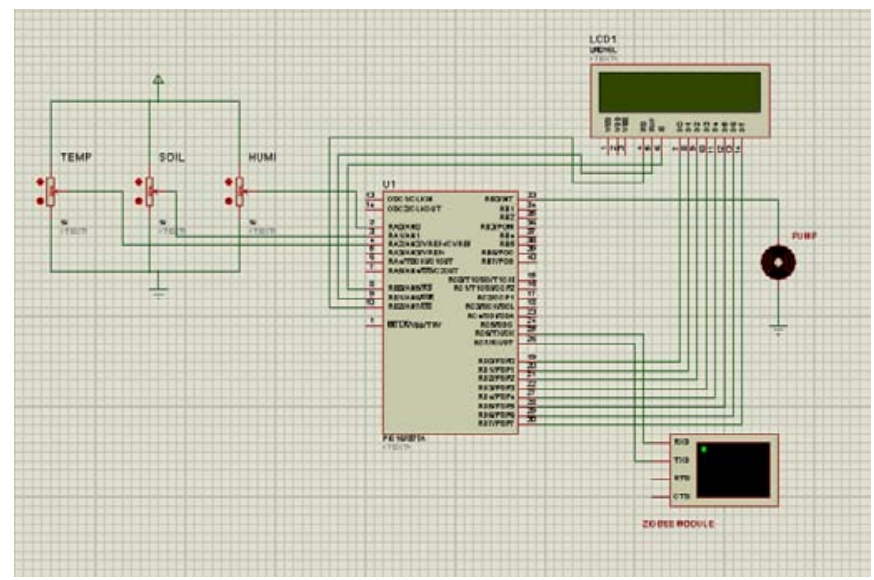

Fig.1, Circuit Diagram

\section{RESUlts AND DISCUSSION}

In this project smart system has been implemented for irrigation system which uses concept of IoT. This project includes various sensors like temperature sensor, humidity sensors, soil moisture sensor. Using communication technology message will be sent to IoT. Thus, here by we conclude that the proposed system removes all the drawbacks of existing system and enhanced with IoT system. The smart irrigation system implemented is cost effective for optimizing water resources for agricultural production. The proposed framework can be utilized to switch on/off the water sprinkler depending on the soil dampness levels in this manner making the prepare easier to utilize. Through this extend it can be concluded that there can be impressive advancement in water system with those of IOT and mechanization. Thus, this system is a solution to the problems faced in the existing process of irrigation.

\section{CONCLUSION AND FUTURE SCOPE}

The utilization of agribusiness arranging development is need of the bleeding edge provincial progression, in like manner yet likewise a basic picture without limits level of agrarian change; it will be the future course of cultivating change. Ensuing to building the cultivating water framework structure equipment and separating and analysing the deal with pecking demand highlights, esteem and the taking a gander at PC program design of exactness agribusiness water framework system, applying the web of things to the exceedingly powerful and secure agrarian generation has a critical effect on 
guaranteeing the productive utilize of water assets as well as guaranteeing the effectiveness and soundness of the rural generation.

Our undertaking can be improvised by including a Webs caper which can foresee the climate and water the plants/edits appropriately. On the off chance that rain is determined, less water is let out for the plants. Additionally, a GSM module can be incorporated so the client can control the framework by means of advanced mobile phone. A water meter can be introduced to evaluate the measure of water utilized for water system and subsequently giving a cost estimation. A solenoid valve can be utilized for fluctuating the volume of water stream. Moreover, Wireless sensors can likewise be utilized.

A. Abbreviations and Acronyms

IoT (Internet of Things), HTTP (Hyper Text Transfer Protocol), GSM (Global System for Mobile communication), GPS (Global Positioning System), GPRS (General Packet Radio Service), DC (Direct Current), LCD (Liquid Crystal Display), IDE (Integrated Development Environment), TCP (Transmission Control Protocol), WSN (Wireless Sensor Network), M2M (Machine to Machine), WPAN (Wireless Personal Area Network), DLL (Data Link Layer), PSU (Personal Supply Unit), CCS (Code Composer Studio), DSP (Digital Signal Processor).

\section{REFERENCES}

[1] Yuthika Shekhar, "Intelligent IoT Based Automated Irrigation System”, International Journal of Applied Engineering Research Volume 12, pp. 7306-7320, 2017.
[2] R. Hemalatha, "Internet of Things (IOT) Based Smart Irrigation”, International Journal of Advanced Research in Biology Engineering Scienvce and Technology, pp.2395695, 2016.

[3] G.V. Satyanarayana, "Wireless Sensor Based Remote Monitoring System for Agriculture Using ZigBee and GPS", Conference on Advances in Communication and Control System, 2013.

[4] Joaquín Gutiérrez, "Automated Irrigation System Using a Wireless Sensor Network and GPRS Module", IEEE Transaction on Instruments and Measurement, pp.00189456, 2013.

[5] A. Sethumathavan, "Automstic Irrigation System using Internet of Things", International Journal for Research in Applied Science \& Engineering Technology, pp.2321-9653, 2016.

[6] A.N Arvindan, "Experimental Investigation of Remote Control Via Android Smart Phone of Arduino-Based Automated Irrigation System

Using Moisture Sensor”, 3rd International Conference on Electrical Energy Systems, Chennai, India, pp.168-174, 2016.

[7] Matti Satish Kumar, "Monitoring moisture of soil using low cost homemade Soil Moisture Sensor and Arduino Uno”,3rd International Conference on Advanced Computing and Communication System(ICACCS-2016), Coimbatore, India, 2016.

[8] Pushkar Singh, “Arduino based smart irrigation Using Water Flow Sensor, Soil Moisture Sensor, Temperature Sensor and ESP8266 Wifi

Module”, Humanitarian Technology Conference (R10-HTC), 2016 IEEE Region 10, Tezpur, India, 2016. 